\title{
Prevalencia de trastornos mentales comunes en barrios marginales urbanos con población desplazada en Colombia
}

\author{
Gloria Puertas, ${ }^{1}$ Cielo Ríos ${ }^{2}$ y Hernán del Valle ${ }^{3}$
}

Forma de citar

Puertas G, Ríos C, Del Valle H. Prevalencia de trastornos mentales comunes en barrios marginales urbanos con población desplazada en Colombia. Rev Panam Salud Publica. 2006;20(5):324-30.

RESUMEN Objetivos. Investigar la prevalencia de trastornos mentales comunes en grupos con un alto porcentaje de familias desplazadas que viven en barrios marginales urbanos en Sincelejo (Sucre), Colombia.

Métodos. Se realizó un estudio transversal con muestreo aleatorio bietápico por conglomerados en barrios considerados marginales de Sincelejo que contienen un alto porcentaje de población desplazada. Se realizó una encuesta domiciliaria para determinar la presencia de trastornos mentales comunes — trastornos psicosomáticos, ansiedad y depresión-en la población estudiada. Se fijó como valor discriminatorio para determinar la presencia de un trastorno mental común en personas de 18 años o más una puntuación de 7 en las 20 primeras preguntas del Cuestionario de declaración de sintomas (Self-Reporting Questionnaire, SRQ) de la Organización Mundial de la Salud. Se buscaron asociaciones entre las variables recogidas (grupo de edad, sexo, tipo de carné sanitario y tiempo de residencia en el barrio), y entre ellas y la puntuación obtenida en el SRQ. Se utilizó la prueba de ji al cuadrado, con un valor de significación estadística de 0,05, y se realizó una regresión logística con todas las variables relacionadas. Resultados. Se encontró una prevalencia de 27,2\% (intervalo de confianza de 95\% [IC95\%]: $24,0 \%$ a 30,0\%) de trastornos mentales comunes en la población adulta de los barrios estudiados. En general, 13,6\% (IC95\%: 11,4\% a 16,1\%) de la población encuestada reconoció tener problemas de consumo excesivo de alcohol. Se encontró una asociación entre la prevalencia de trastornos mentales comunes y el tipo de carné usado para acceder a la atención de salud (razón de posibilidades [RP]: 1,66 para el carné de desplazado frente a cualquiera de los otros dos) y el sexo (RP: 1,78 para mujeres frente a hombres); dicha asociación persistió aun después del ajuste en función de las demás variables explicativas.

Conclusión. La prevalencia de trastornos mentales comunes en las poblaciones urbanas valoradas mostró una asociación estadística con el tipo de carné, del cual depende a su vez el acceso a los servicios públicos de salud. Dicha asociación es lo suficientemente alta para justificar la prestación de cuidados de salud mental a esta población. Aunque no se consiguió identificar adecuadamente la población desplazada en el grupo estudiado $y$, por tanto, medir en ella con el SRQ la prevalencia puntual de trastornos mentales comunes, los diferentes sistemas de acceso a la salud en Colombia, que contemplan un acceso determinado para los desplazados, parecen asociarse con la prevalencia de estos trastornos en barrios periféricos urbanos.

Palabras clave Salud mental, poblaciones vulnerables, Colombia.

Coordinadora regional de salud del Alto Comisionado para los Refugiados (ACNUR) para el sur de África. Toda correspondencia debe dirigirse a esta autora a: C/ Pedro IV n ${ }^{\circ} 68,2^{\circ}$ Derecha. 22002
Huesca, España. Correo electrónico: puertas@ unhcr.org

2 Coordinadora Médica de Médicos sin Fronteras Holanda, Harare, Zimbabwe.
Departamento de Asuntos Humanitarios de Médicos sin Fronteras Holanda, Ámsterdam, Países Bajos. 
Varias décadas de conflicto armado e inestabilidad política en Colombia han obligado a millones de personas a huir de sus hogares; diferentes estimaciones valoran entre 1,4 (1) y 2,5 millones (2) el número de desplazados dentro del país hasta junio de 2004. Todavía muchos desplazados internos permanecen en áreas inaccesibles a las organizaciones humanitarias, pero la tendencia de los últimos cinco años ha sido el incremento de los desplazados en las afueras de las grandes ciudades. Si bien este desplazamiento no se da en masa, sino de forma individual o familiar, datos preliminares indican que hay grandes bolsas de personas desplazadas que viven en condiciones precarias en los suburbios urbanos. Una de las ciudades en Colombia que alberga un gran número de desplazados es Sincelejo, en el departamento de Sucre.

Médicos sin Fronteras Holanda (MSFH) ha trabajado con poblaciones afectadas por el conflicto armado en zonas rurales del norte de Colombia desde 1997. En octubre de 2004, esta organización decidió ampliar su asistencia sanitaria a las poblaciones afectadas que viven en zonas urbanas $y$, a tal efecto, se valoraron dos localidades del norte colombiano: Santa Marta (Magdalena) y Sincelejo.

Las enfermedades mentales constituyen una carga importante para la sociedad colombiana, como lo puso de manifiesto el estudio llevado a cabo por la Organización Panamericana de la Salud (OPS) y el Ministerio de Protección Social en el año 2003 (3), en el cual se encontró una prevalencia de cualquier trastorno de salud mental de $16,0 \%$ en un período de un año y de $7,6 \%$ si se consideraban los últimos 30 días. En otros estudios (4) que describen perfiles epidemiológicos de la población desplazada en Colombia se han encontrado mayores niveles de estrés mental en esta población que en otras, de una forma constante en cuatro localidades diferentes (Cartagena, Montería, Cali y Soacha) (5).

Teniendo en cuenta lo anteriormente expuesto y como parte de la metodología de evaluación de las necesidades sanitarias en esos barrios marginales que albergan desplazados, se llevó a cabo una encuesta de salud mental con el propósito de estimar la prevalencia de trastornos mentales comunes (TMC). Los trastornos comunes de salud mental abarcan la depresión, la ansiedad y los trastornos psicosomáticos; el término engloba los trastornos psicológicos denominados «trastornos neuróticos, somatomorfos $\mathrm{y}$ relacionados con el estrés» y los «trastornos del estado de ánimo» según la décima edición de la Clasificación internacional de enfermedades (CIE-10). El término se ha utilizado en diferentes publicaciones $(6,7)$ y, según un estudio multicéntrico en varios países (8), los TMC representan alrededor de $24,0 \%$ de la morbilidad observada en los servicios de atención primaria de salud de la población en general.

El propósito de este artículo es presentar los resultados de la encuesta de salud mental realizada en los barrios periféricos de Sincelejo, donde vive una gran cantidad de población desplazada procedente de otras partes del departamento de Sucre y de los departamentos circundantes, usando el Cuestionario de declaración de síntomas (Self-Reporting Questionnaire, SRQ) de la Organización Mundial de la Salud (OMS) (9). Este cuestionario ha sido ampliamente utilizado para estimar la prevalencia puntual de TMC.

\section{MATERIALES Y MÉTODOS}

La población objeto de estudio fueron las personas desplazadas. Sin embargo, la definición de «desplazado» en este contexto no es obvia. Las familias que se desplazan debido al conflicto armado en Colombia suelen registrarse como desplazados en la Red de Solidaridad Social para acceder a una ayuda de emergencia de hasta seis meses de duración y reciben un «carné de desplazado». Este carné permite también el acceso al sistema público de salud siempre y cuando la familia no disponga de otro carné sanitario. No es el objetivo de este artículo describir el sistema sanitario colombiano, pero para acceder a él es necesario tener un «carné de usuario», como se describirá más adelante. El «carné de desplazado» no es el mejor criterio para identificar a una familia como desplazada, ya que muchas personas que están en situación de desplazamiento acceden a la atención de salud con carnés diferentes que garantizan una mejor atención. Por otra parte, encuestar solamente a desplazados en este contexto tan particular hubiese sido cuestionable desde el punto de vista ético. Por ello se incluyeron en la encuesta todos los barrios de Sincelejo conocidos por albergar población desplazada y a todos sus habitantes.

Algunas organizaciones no gubernamentales (ONG) y gubernamentales, así como personas clave que trabajan en estos barrios marginales, identificaron 13 barrios que albergaban desplazados. Se visitaron todos estos barrios para confirmar la presencia de población desplazada antes de realizar la encuesta. Las cifras de población de cada barrio se obtuvieron de la Secretaría de Planeación de Sincelejo y se cotejaron con líderes comunitarios y con un censo reciente realizado por una ONG (Plan Internacional).

La distribución de las casas en los barrios escogidos para la encuesta era demasiado errática y dispersa, sin calles bien definidas, lo que impidió realizar una encuesta sistemática. Por ello se realizó un muestreo por conglomerados. Según este método, cuando la prevalencia se desconoce y se busca un nivel de significación estadística de $95 \%$, es necesario realizar un mínimo de 768 encuestas (10) en 30 conglomerados. Se estimó la población acumulada de todos los barrios del estudio, se escogieron 30 conglomerados al azar y se calculó el intervalo de muestreo (población total que se buscaba encuestar dividida entre el número de conglomerados). En cada conglomerado se eligieron aleatoriamente 30 casas.

Se entrenó en el uso del cuestionario SRQ a tres miembros del equipo de MSFH y a 12 estudiantes de la Facultad de Psicología de la Corporación Universitaria del Caribe (CECAR) de Sincelejo. Este cuestionario consta de 30 preguntas de respuesta dicotómica — sí o no-y lo diseñó la Organización Mundial de la Salud como instru- 
mento de cribado de trastornos mentales en el contexto de la atención primaria de salud para estimar la prevalencia puntual de estas afecciones. Las primeras 20 preguntas hacen referencia a síntomas somáticos y psicológicos y las 10 restantes buscan la presencia de síntomas psicóticos y de problemas relacionados con el consumo de alcohol. El período de tiempo tomado en consideración fueron los últimos 30 días. Si bien la OPS ya ha validado el SRQ y varias ONG lo han utilizado en Colombia, aún no se ha fijado el valor discriminatorio adecuado para definir los trastornos mentales comunes en el país. En este estudio se optó por el valor discriminatorio de 7, utilizado en algunos estudios previos $(11,12)$, aunque diferente del utilizado en otros estudios (13), con el fin de identificar a las personas con trastornos comunes de salud mental. La encuesta se realizó durante los días 28 y 29 de octubre de 2004. Se comenzaban las encuestas en el centro de cada conglomerado, siguiendo una dirección cualquiera y avanzando hacia la periferia. Si un domicilio estaba vacío, se pasaba al siguiente. Los moradores de todos los domicilios habitados accedieron a responder al cuestionario. Se seleccionó al azar una persona adulta en cada domicilio.

Además de las 30 preguntas del SRQ, se añadieron otras de tipo sociodemográfico: sexo de la persona entrevistada, edad, tiempo transcurrido desde la llegada al barrio y tipo de carné de acceso al sistema público de salud. El acceso a la atención de salud se consideró una variable importante y se decidió medirlo de acuerdo con el tipo de carné sanitario utilizado por las familias. Se contemplaron cuatro posibilidades: carné de desplazado, del Sistema de Identificación de Beneficiarios (SISBEN), de las Administradoras del Régimen Subsidiado (ARS) y ninguno. Solo el carné de tipo ARS permite el acceso a la mayoría de los servicios sanitarios. El carné de desplazado y el del SISBEN permiten obtener cuidados de urgencia, pero el carné del SISBEN es el paso previo a la obtención del carné de las ARS. Los pacientes sin ningún carné tienen que pagar para recibir cualquier tipo de atención de salud.

Como medida adicional para definir mejor la población desplazada, además del carné correspondiente se incluyó una pregunta sobre la duración de la estancia en el barrio (tiempo transcurrido desde la llegada). Esta variable temporal podría considerarse además como un indicador de vulnerabilidad, ya que las familias llegadas más recientemente presentan características de fragilidad diferentes a las de hogares asentados durante más tiempo. El análisis estadístico se realizó con la versión 8.0 de Stata. Se buscaron asociaciones estadísticamente significativas entre las diferentes variables sociodemográficas descritas anteriormente, entre cada una de ellas y la puntuación obtenida en el cuestionario, entre cada una de ellas y el consumo excesivo de alcohol autodeclarado, y entre este último y la puntuación del cuestionario, mediante la prueba de ji al cuadrado. Se calcularon las razones de posibilidades (RP) estadísticamente significativas. Posteriormente se construyeron modelos con varias variables y se realizó una regresión logística, observando el valor de la razón de posibilidades y el resultado de la prueba del cociente de verosimilitudes.

\section{RESULTADOS}

Se entrevistó a un total de 904 personas. Tras realizar la limpieza de la base de datos para eliminar del análisis los cuestionarios con datos incompletos, se incluyeron 878 encuestas en el análisis; $32 \%$ de la población encuestada fueron hombres y $68 \%$ mujeres. La edad se estratificó en cuatro categorías: entre 18 y 25 años, entre 26 y 45 años, entre 46 y 65 años y mayores de 65 años. Así, 24,7\% de los encuestados tenían menos de 25 años; $51,8 \%$ pertenecían al grupo de 26 a 45 años; $18,4 \%$ al de 46 a 65 años; y 5,0\% al de mayores de 65 años. Con respecto al tiempo de permanencia en el barrio, 16,7\% había llegado hacía menos de un año; $40,4 \%$, hacía de 1 a 5 años; y 42,0\%, hacía 6 años o más. En términos del tipo de carné sanitario, $16,9 \%$ de los encuestados no tenía ninguno; 22,9\% utilizaba el carné de desplazado; 32,9\% utilizaba el carné del SISBEN; y 27,1\% tenía el carné de las ARS.

Se encontró una prevalencia general de TMC de 27,2\% (intervalo de confianza de $95 \%$ [IC95\%]: $24,0 \%$ a 30,0\%). Esta prevalencia fue de $31,0 \%$ (IC95\%: $27,0 \%-34,0 \%$ ) en las mujeres y de 20,0\% (IC95\%: $15,0 \%$ a $25,0 \%$ ) en los hombres (RP: 1,$78 ; P<0,001)$. La prevalencia de TMC aumentó con la edad y fue de $20,7 \%$ en el grupo de edad de 18 a 25 años (IC95\%: 15,5\% a 26,7\%), de $26,4 \%$ (IC95\%: $22,0 \%$ a $30,6 \%$ ) en el grupo de 26 a 45 años, de 35,2\% (IC95\%: 28,0\% a 43,0\%) en el grupo de 46 a 65 años, y de 38,6\% (IC95\%: 24,3\% a 54,0\%) en los mayores de 65 años; las diferencias entre estos grupos de edad fueron estadísticamente significativas $(P=0,005)$. El tiempo de permanencia en el barrio no estuvo asociado con la prevalencia de TMC: 26,0\% (IC95\%: 19,6\% a 34,0\%) en el grupo de personas que había llegado al barrio hacía menos de un año; 30,0\% (IC95\%: 20,0\% a 41,0\%) en el grupo llegado hacía 1 a 2 años; 33,0\% (IC95\%: $24,0 \%$ a $44,0 \%$ ) en el llegado hacía 3 años; $23,0 \%$ (IC95\%: $15,0 \%$ a 32,0\%) en el llegado hacía 4 años; $29,0 \%$ (IC95\%: 20,0\% a 41,0\%) en el llegado hacía 5 años; y 26,0\% (IC95\%: 22,0\% a $31,0 \%$ ) en el llegado hacía 6 años o más. Todas las RP se presentan en el cuadro 1. Para la edad, la RP se calculó mediante una aproximación por cada unidad de aumento de edad. El grupo de edad de 18 a 25 años tuvo valor 1 , el siguiente, 2, el siguiente, 3 y el de mayores de 65 años, 4 .

La duración de la estancia en el barrio se asoció con el tipo de carné sanitario. Los recién llegados (permanencia inferior a un año) tenían cada tipo de carné en una proporción similar y 23,0\% de ellos no tenían ningún carné. Tras 6 años de residencia en el barrio, la mayoría de las familias habían obtenido el carné SISBEN $(35,0 \%)$ o el carné ARS (40,0\%); en cambio, 12,0\% seguía usando el carné de desplazado y 13,6\% carecía de carné. Todas estas diferencias fueron estadísticamente significativas $(P<0,001)$. 
De todos los encuestados, $11,3 \%$ (IC95\%: 9,3\% a 13,5\%) reconoció haber tenido pensamientos suicidas en los 30 días anteriores a la encuesta y de este último grupo, 70,0\% presentó una puntuación positiva en relación con los TMC (IC95\%: 60\% a 78\%; $P<0,001$ ). En función de la antigüedad en el barrio, $16,3 \%$ de los recién llegados admitieron haber tenido pensamientos suicidas, en comparación con solo 10,3\% de los que tenían más de un año de antigüedad $(P=0,03)$. El sexo y la edad no se asociaron con la presencia de pensamientos suicidas.

En general 13,6\% de los encuestados reconoció haber tenido algún tipo de problema relacionado con el consumo de alcohol en los últimos 30 días, (IC95\%: $11,4 \%$ a $16,1 \%$ ) y las diferencias según el sexo fueron estadísticamente significativas: $29,0 \%$ (IC95\%: $23,5 \%$ a $34,5 \%$ ) de los hombres y $6,4 \%$ (IC95\%: 4,5\% a 8,6\%) de las mujeres $(P<0,001)$. Sin embargo, los problemas con el consumo de alcohol no se relacionaron con los TMC según el cuestionario, incluso después de ajustar por el sexo y la edad, posibles factores de confusión. El consumo de alcohol sí se relacionó con la duración de la permanencia en el barrio: su frecuencia fue de $19,2 \%$ en los recién llegados y de $12,3 \%$ en quienes habían llegado hacía más de un año $(P=0,03)$.

El tipo de carné sanitario utilizado por las familias se asoció discretamente con la prevalencia de TMC y también con una respuesta afirmativa a la pregunta sobre pensamientos suicidas y a cualquiera de las preguntas sobre el consumo de alcohol (cuadro 2).

Debido a las dificultades anteriormente mencionadas para definir la población desplazada y considerando los resultados del cuadro 2, se categorizó de nuevo la variable tipo de carné en una variable binaria - carné de desplazado o cualquiera de los otros-en un intento por medir más específicamente los TMC entre los desplazados. Con esta reorganización de los datos, la RP bruta de los TMC en los usuarios del carné de desplazados fue de 1,70 (IC95\%: 1,21 a 2,$39 ; P=0,002$ ), frente al grupo de referencia de usuarios con cualquier otro tipo de carné.

CUADRO 1. Razón de posibilidades bruta de los trastornos mentales comunes, por sexo, edad y duración de la estancia en el barrio nuevo. Sincelejo (Sucre), Colombia, octubre de 2004

\begin{tabular}{lccc}
\hline & RPa bruta & IC95\% ${ }^{\text {b }}$ & $P$ \\
\hline Sexo & 1,78 & 1,26 a 2,50 & 0,0009 \\
Edad & 1,40 & 1,16 a 1,69 & 0,0004 \\
Tiempo de estancia & 0,96 & 0,79 a 1,18 & 0,73 \\
\hline
\end{tabular}

a $\mathrm{RP}$ : razón de posibilidades.

IC95\%; intervalo de confianza del $95 \%$.

CUADRO 2. Prevalencia de trastornos mentales comunes (TMC), pensamientos suicidas y consumo excesivo de alcohol autodeclarado, según el tipo de carné sanitario. Sincelejo (Sucre), Colombia, octubre de 2004

\begin{tabular}{lccccc}
\hline & $\begin{array}{c}\text { Sin carné } \\
\%\end{array}$ & $\begin{array}{c}\text { Desplazado } \\
\%\end{array}$ & $\begin{array}{c}\text { SISBEN } \\
\%\end{array}$ & $\begin{array}{c}\text { ARS }^{b} \\
\%\end{array}$ & \\
\hline $\begin{array}{l}\text { Puntuación positiva en relación } \\
\quad \text { con los TMC }\end{array}$ & 24,2 & 35,8 & 25,3 & 24,0 & $\begin{array}{c}\chi^{2}=12,73 \\
P=0,01\end{array}$ \\
Pensamientos suicidas (Sí) & 6,7 & 16,5 & 8,3 & 13,4 & $\begin{array}{c}\chi^{2}=12,18 \\
P=0,016\end{array}$ \\
Consumo excesivo de alcohol (Sí) & 16,3 & 8,5 & 12,6 & 16,8 & $\begin{array}{c}\chi^{2}=14,21 \\
P=0,007\end{array}$ \\
& & & & &
\end{tabular}

a SISBEN: Sistema de Identificación de Beneficiarios.

${ }^{b}$ ARS: Administradoras del Régimen Subsidiado.

Para eliminar el efecto de los conglomerados en el diseño del muestreo, se realizó un análisis de regresión logística mediante un ajuste con respecto a este factor e incluyendo en el modelo las variables sexo, edad, tiempo de permanencia en el barrio y tipo de carné (variable binaria). Se planteó como hipótesis que dos variables - TMC y consumo de alcohol- podrían estar asociadas con la variable "tipo de carné para acceder a la atención de salud", después de ajustar por las variables de confusión. El cuadro 3 describe estos datos.

Se siguió observando una asociación entre la prevalencia de TMC y el tipo de carné y el sexo aun después de hacer ajustes para compensar el posible efecto de confusión de las otras variables explicativas (sexo, edad y tiempo de permanencia en el barrio). La asociación de la variable sexo con el consumo excesivo de alcohol también se mantuvo presente.

Para discernir el efecto del diseño por conglomerados utilizado en el muestreo, se realizaron análisis de la varianza dentro de cada conglomerado y entre los distintos conglomerados. La varianza dentro de cada conglomerado fue mayor que la observada entre conglomerados $(F=2,66$; con 29 grados de libertad; $P<0,0001$ ); el hecho de pertenecer a un conglomerado concreto no se asoció con la prevalencia de TMC.

\section{DISCUSIÓN}

En este estudio se ha tratado de estimar la prevalencia de TMC en las familias desplazadas en un contexto urbano (Sincelejo [Sucre], Colombia), pero el objetivo se vio dificultado por varios factores. El primero fue la dificultad de realizar la encuesta solo en la población desplazada, ya que esta se encontraba mezclada con la población no desplazada que vive en los suburbios. Se puede considerar que las personas que viven en este tipo de barrios tienen características socioeconómicas similares. Sin embargo, el desplaza- 
CUADRO 3. Valores de las razones de posibilidades, ajustadas por la prevalencia de trastornos mentales comunes y del consumo excesivo de alcohol autodeclarado, según el tipo de carné y el sexo. Sincelejo (Sucre), Colombia, octubre de 2004

\begin{tabular}{|c|c|c|c|c|}
\hline \multirow[b]{2}{*}{ Regresión logística } & \multicolumn{2}{|c|}{ Trastornos mentales comunes } & \multicolumn{2}{|r|}{ Alcohol } \\
\hline & $\mathrm{RP}^{\mathrm{a}}$ & $(\mathrm{IC} 95 \%)^{\mathrm{b}} ; P$ & RP & (IC95\%); $P$ \\
\hline \multicolumn{5}{|l|}{ Tipo de carnéc } \\
\hline $\begin{array}{l}\text { De desplazado } \\
\text { Otro tipo (referencia) }\end{array}$ & $\begin{array}{l}1,66 \\
1,0\end{array}$ & $(1,16$ a, 52$) ; 0,003$ & 0,60 & $(0,34$ a 1,08$) ; 0,09$ \\
\hline \multicolumn{5}{|l|}{$\operatorname{Sexo}^{d}$} \\
\hline $\begin{array}{l}\text { Mujer } \\
\text { Hombre (referencia) }\end{array}$ & $\begin{array}{l}1,78 \\
1,0\end{array}$ & $(1,25$ a 2,51$) ; 0,001$ & 0,17 & $(0,11$ a 0,26$) ;<0,0010$ \\
\hline $\mathrm{CV}^{\mathrm{e}}$ & 33,32 & & 91,47 & \\
\hline
\end{tabular}

a RP: Razón de posibilidades (odds ratio).

${ }^{b}$ IC95\%: intervalo de confianza del $95 \%$.

c Tratado como variable binaria y ajustado según el sexo, la edad y la duración de la estancia en el barrio nuevo.

d Ajustado según la edad, el tipo de carné y la duración de la estancia en el barrio nuevo.

e CV: cociente de verosimilitudes.

miento en sí mismo puede ser un factor estresante con un impacto sobre la salud mental que no pudo medirse adecuadamente. Se utilizaron dos variables como aproximación a la situación de desplazado, la duración de la estancia en el nuevo barrio y la posesión de un carné de desplazado, aunque ambas entrañan limitaciones que impidieron identificar a los desplazados con exactitud. La duración de la estancia en el nuevo barrio se aplicó como criterio de vulnerabilidad, ya que las personas recién llegadas suelen ser las más duramente afectadas por el desplazamiento. Por otra parte, el carné de desplazado deberían poseerlo sólo las personas desplazadas, por definición, pero en realidad hay otras que lo han obtenido para conseguir comida, otros productos y acceso a la atención de salud de urgencia gratuita. Asimismo, algunas personas desplazadas han conseguido otros carnés que les garantizan mejor acceso a los servicios de salud.

Una segunda limitación del estudio fue el método de muestreo por conglomerados, que no permite establecer comparaciones entre los mismos porque los barrios con altos porcentajes de desplazados entre sus moradores están mezclados en el análisis con barrios que tienen proporciones inferiores. Desafortunadamente, ni los diferentes barrios ni los conglomerados extraídos de diferentes barrios pueden compararse entre sí; cabe afirmar, como máximo, que la muestra es representativa de los 13 barrios evaluados, pues el tamaño muestral y el método de muestreo (por conglomerados, bietápico y aleatorio) garantizan la representatividad.

Una tercera limitación es la sobrerrepresentación de mujeres en la muestra. Como la encuesta se realizó durante la mañana y las primeras horas de la tarde, la mayoría de los hombres estaban ausentes de los hogares, lo cual disminuyó la probabilidad de que los encuestados fueran hombres. No obstante, la diferencia en la prevalencia de TMC entre hombres y mujeres fue estadísticamente significativa $(P=0,001)$ y se mantuvo aun después de hacer ajustes en función de otras variables explicativas, como la edad, el tipo de carné sanitario y el tiempo de permanencia en el nuevo barrio.

Pese a las citadas limitaciones, uno de los hallazgos principales es que la prevalencia de TMC observada en esta encuesta fue mayor que la hallada en el Estudio Nacional de Salud Mental. En estos dos estudios se utilizaron dos instrumentos de medida muy diferentes; en el Estudio Nacional de Salud Mental se utilizó la Entrevista Diagnóstica Internacional Compuesta (CIDI, por el inglés Composite International Diagnostic Interview); sin embargo, ambos instrumentos han demostrado su validez para medir los TMC en di- ferentes contextos (14). Esta diferencia metodológica limita la comparación de los resultados de ambas encuestas, pero la discrepancia encontrada sigue siendo interesante. En el Estudio Nacional de Salud Mental se encontró una prevalencia de $7,4 \%$ de «cualquier trastorno de salud mental» en los 30 días anteriores a la encuesta en una muestra de adultos (mayores de 18 años) que vivían en ciudades con hogares estables. En nuestro estudio, realizado también en un contexto urbano pero dirigido hacia barrios desfavorecidos desde el punto de vista socioeconómico, se encontró una prevalencia bastante más alta de TMC de 27,6\% en un grupo de edad similar (mayores de 18 años). Una condición socioeconómica baja se ha asociado con morbilidad psiquiátrica (15), discapacidad, acceso deficiente a los cuidados de salud y un pronóstico desfavorable de salud (16). Asimismo, la depresión se ha mostrado asociada con la baja condición socioeconómica (17). En esta encuesta, realizada en barrios urbanos de condición socioeconómica baja, se ha encontrado una prevalencia de TMC mayor que en otro estudio realizado también en una zona urbana, pero sin tener en cuenta la situación socioeconómica de las personas estudiadas. Sin embargo, las diferencias metodológicas antes mencionadas no permiten profundizar en las razones de esta discrepancia. Se precisan más investigaciones en Colombia a fin de estudiar las relaciones entre algunas variables socioeconómicas y la presencia de trastornos mentales en contextos rurales y urbanos.

Como hallazgo constante en las encuestas de salud mental en la población en general, la tasa de prevalencia de trastornos depresivos es mayor en las mujeres que en los hombres. En una revisión exhaustiva (18) se encontró que en estudios realizados en la población en general, las mujeres presentaban a lo largo de la vida tasas de prevalencia de depresión mayor, más altas que los hombres. En algunos países en desarrollo, entre ellos Chile y la India, las tasas de prevalencia de TMC más altas se han asociado con el sexo femenino, la poca educación y la pobreza (19). En el 
estudio colombiano de salud mental se encontraron también tasas más altas de depresión en las mujeres que en los hombres. El presente estudio aporta nuevas pruebas a favor de una asociación entre el sexo y la presencia de TMC en un país en desarrollo.

Sorprendentemente, los recién llegados a los barrios estudiados (menos de un año) y los vecinos asentados durante períodos más prolongados mostraron puntuación positivas semejantes del SRQ en relación con los TMC. Estos recién llegados podrían considerarse el grupo más cercano al de la población desplazada, que fue la población de interés en el presente estudio. Sin embargo, la encuesta no reveló una prevalencia más alta de TMC entre los recién llegados, a pesar de tener un acceso precario a la atención de salud (como lo demuestra el tipo de carné utilizado) y de presentar puntuaciones más altas en el SRQ en relación con pensamientos suicidas y un consumo excesivo de alcohol. El impacto de la migración sobre la salud mental se ha estudiado repetidamente y las explicaciones dadas en diferentes fuentes todavía son objeto de debate $(20,21)$. En este estudio no se encontró ninguna asociación entre el tiempo transcurrido desde el cambio de lugar de residencia y la prevalencia puntual de TMC.

Las diferencias encontradas con respecto al tipo de carné sanitario utilizado son difíciles de interpretar. En la población entrevistada, las personas sin ningún tipo de carné (y que por lo tanto tenían que pagar los cuidados de salud recibidos) no fueron diferentes de las personas con un mejor acceso a la atención de salud (poseedores de carné ARS) con respecto a la prevalen- cia de TMC y al consumo excesivo de alcohol. Sin embargo, los usuarios del carné de desplazados difirieron considerablemente del resto, ya que tuvieron una prevalencia mayor de TMC. Esta diferencia persistió incluso después de hacer ajustes en función del efecto de otras variables explicativas (sexo, edad y duración de la estancia en el barrio nuevo). Teóricamente, si en la zona estudiada se lleva un buen registro de las personas desplazadas, cualquiera que posee un carné de desplazado debe serlo en realidad. Sin embargo, también hay personas desplazadas que tienen otros tipos de carné, las cuales constituyen un grupo bastante heterogéneo de personas con diferente acceso a los servicios de salud. ¿Qué características especiales tienen los usuarios del carné de desplazado? No lo sabemos. Para averiguarlo sería necesario un análisis más detallado, utilizando métodos cualitativos, de las actitudes y creencias de la población colombiana en torno al sistema de los carnés sanitarios. No puede afirmarse que poseer un carné de desplazado para acceder al sistema público de salud tenga una influencia en la prevalencia de TMC. Sin embargo, las razones por las cuales las personas que tienen este carné difieren del resto con respecto a su estado de salud mental, según la puntuación obtenida en el SRQ, podrían estar relacionadas con sus mecanismos de afrontamiento, su temor frente al procedimiento de registro como desplazados, las complejidades del sistema de estratificación del SISBEN y muchos otros factores que sobrepasan la información recogida en este estudio y el objetivo del mismo.
Si bien este estudio contribuye a aumentar los conocimientos acerca de la prevalencia de TMC en barrios pobres con altas proporciones de población desplazada en Sincelejo, se precisa realizar más investigaciones con el fin de valorar la prevalencia de estos trastornos en la población desplazada en Colombia. En el momento de elaborar políticas de salud, habría que comparar la frecuencia de trastornos mentales en la población desplazada y la población no desplazada, en contextos rurales y urbanos. La prevalencia de TMC en los barrios estudiados en este ambiente urbano es lo suficientemente alta para justificar la prestación de servicios adecuados de salud mental. Aunque no se consiguió identificar adecuadamente la población desplazada en el grupo estudiado y, por tanto, medir en ella con el SRQ la prevalencia puntual de trastornos mentales comunes, los diferentes sistemas de acceso a la salud en Colombia, que contemplan un acceso específico y delimitado para los desplazados con la consiguiente prestación de servicios de salud, parecen asociarse con la prevalencia de estos trastornos en barrios periféricos urbanos.

Agradecimiento. La autora principal les agradece a Vikram Patel su apoyo y útiles comentarios en el análisis de los datos y borradores previos y a Larraitz Arriola su ayuda en la interpretación de los resultados estadísticos. El equipo de Médicos sin Fronteras Holanda que realizó la encuesta en Sincelejo agradece también el entusiasmo de los estudiantes de la Universidad del CECAR en Sincelejo que participaron en la encuesta.

\section{REFERENCIAS}

1. Red de Solidaridad Social (RSS). Sistema único de registro de población desplazada por la violencia en Colombia. Hallado en www. red.gov.co. Acceso el 13 de octubre de 2004.

2. Consultoría para los derechos humanos y el desplazamiento (CODHES). Desplazados: ni seguridad ni democracia. Codhes Informa. 2004;(47): 1-17. Hallado en: http://www. codhes.org/Web/Info/Boletines/Boletin47. pdf. Acceso el 14 de octubre de 2004.
3. Colombia, Ministerio de la Protección Social. Un panorama nacional de la salud y enfermedad mental en Colombia: informe preliminar. Estudio Nacional de Salud Mental. Santa Fe de Bogotá: MPS; 2003.

4. Cáceres D, Izquierdo V, Mantilla L, Blandía M, Gonzáles A, Jara J. Perfil epidemiológico de la población desplazada por el conflicto armado interno del país en un barrio de la ciudad de Cartagena, Bolívar, y propuesta de un sistema de vigilancia para la población afectada por este fenómeno en Colombia. Santa Fe de Bogotá: Ministerio de Salud, Instituto Nacional de Salud; 2001.

5. Organización Panamericana de la Salud, Instituto Nacional de Salud. Perfil epidemiológico de la población desplazada y de la población estrato uno no desplazada en cuatro ciudades de Colombia. Santa Fe de Bogotá: OPS; 2003. 
6. Araya R, Rojas G, Lewis G. Common mental disorders in Santiago, Chile. Prevalence and socio-demographic correlates. Br J Psychiatry. 2001;178:228-33.

7. Patel V, Chisolm D, Rabe-Hesketh S, DiasSaxena F, Andrew Gracy, Mann A. Efficacy and cost-effectiveness of drug and psychological treatments for common mental disorders in general health care in Goa, India: a randomised control trial. Lancet. 2003;361:33-9.

8. Ustun T, Sartorius N, eds. Mental illness in general health care: an international study. Chichester (U.K.): John Wiley \& Sons on behalf of WHO; 1995.

9. World Health Organization. A user's guide to the Self-Reporting Questionnaire (SRQ). WHO/MNH/PSF/94.8. Geneva: World Health Organization; 1994.

10. Boelaert M, Davis A, Le Lin B, Michelet M, Ritmeijer K, Van Der Kam S et al. Nutritional guidelines. (First edition). Paris: Médecins sans Frontières; 1998.

11. Harding TW, de Arango MV, Baltazar J, Climent CE, Ibrahim HH, Ladrido-Ignacio L. Mental disorders in primary health care: a study of their frequency and diagnosis in four developing countries. Psychol Med. 1980;2: 231-41.

12. Al-Lawati J, Al-Lawati N, Al-Siddiqi $\mathrm{M}$, Antony SX, Al-Naamani A, Martin RG, et al. Psychological morbidity in primary health care in Oman. A preliminary study. Medical Sciences. 2000;2:105-10.

13. Penayo U, Kullgren G, Caldera T. Mental disorders amongst primary health care patients in Nicaragua. Acta Psychiatr Scand. 1990; 82(1):82-5.

14. Lewis G, Pelosi AJ, Araya R, Dunn G. Measuring psychiatric disorders in the community: a standardised assessment for use by lay interviewers. Psychol Med. 1992;22:465-86.

15. Andrade L, Caraveo-Anduaga JJ, Berglund P. Cross-national comparisons of the prevalences and correlates of mental disorders. Bull World Health Organ. 2000;78:413-26.

16. Weich S, Lewis G. Poverty, unemployment, and common mental disorders: population based cohort study. BMJ. 1998;317:115-9.

17. Lorant V, Deliège D, Eaton W, Robert A, Philippot P, Ansseau M. Socioeconomic in- equalities in depression: a meta-analysis. Am J Epidemiol. 2003;157:98-112.

18. Piccinelli M, Gomez F. Gender differences in the epidemiology of affective disorders and schizophrenia. Geneva: World Health Organization, Division of Mental Health and Prevention of Substance Abuse; 1997.

19. Patel V, Araya R, de Lima MS, Ludermir A, Todd C. Women, poverty and common mental disorders in four restructuring societies. Soc Sci Med. 1999;49:1461-71.

20. Fullilove MT. Psychiatric implications of displacement: contributions from the psychology of place. Am J Psychiatry. 1996;153(12):1516-23.

21. Mumford DB, Minhas FA, Akhtar I, Akhter S, Mubbashar MH. Stress and psychiatric disorders in urban Rawalpindi. Community Survey. Br J Psychiatry. 2000;177:557-62.

Manuscrito recibido el 18 de mayo de 2005. Aceptado para publicación, tras revisión, el 4 de mayo de 2006.
ABSTRACT

The prevalence of common mental disorders in urban slums with displaced persons in Colombia

Key words Mental health, refugees, violence, Colombia.
Objectives. To investigate the prevalence of common mental disorders in groups with a high percentage of families who have been displaced by armed conflict and political instability and are living in urban slum areas on the outskirts of Sincelejo, a city in the department of Sucre, Colombia.

Methods. A cross-sectional study was conducted, with two-stage random cluster sampling, in slum neighborhoods of Sincelejo that contain a high percentage of displaced persons. A household survey of persons 18 years old or older was used to determine the presence of common mental disorders-psychosomatic disorders, anxiety, and depression-in the population studied. A score of 7 or more on the first 20 questions of the Self-Reporting Questionnaire (SRQ) of the World Health Organization was used to determine the presence of a common mental disorder. We searched for associations among the studied variables (age group, gender, type of health care card, and length of residence in the neighborhood) and among those variables and the score on the SRQ. The chi-square test was used, with statistical significance set at 0.05 . Logistic regression was carried out with all the related variables.

Results. The prevalence of common mental disorders in the adult population of the neighborhoods studied was $27.2 \%$ (95\% confidence interval $(95 \% \mathrm{CI})=24.0 \%$ to $30.0 \%$ ); $13.6 \%$ ( $95 \% \mathrm{CI}=11.4 \%$ to $16.1 \%$ ) of the population surveyed had problems with excessive alcohol consumption. There was an association between the prevalence of common mental disorders and the type of health care card used (odds ratio $=1.66$ for persons using the health care card for displaced persons versus persons using other types of health care cards). In addition, there was an association between the prevalence of common mental disorders and gender ( $\mathrm{OR}=1.78$ for women); this association remained after adjusting for other explanatory variables.

Conclusions. Using the study criteria (type of health care card and length of time living in the slum area), it was difficult to separate the displaced persons from other persons living in the same slum areas, and thus to measure the prevalence of common mental disorders among just the displaced persons. The prevalence of common mental disorders in the urban population studied showed a statistical association with the type of health care card, which in turn determined the level of access to public health services. This association between the type of health care card held and the prevalence of common mental disorders is strong enough to justify providing mental health care services to persons who have the health care card for displaced persons. 